\section{Ultrasonography of acute flank pain: a focus on renal stones and acute pyelonephritis}

\author{
Ki Choon Sim
}

Department of Radiology, Korea University Anam Hospital, Korea University College of Medicine, Seoul, Korea

Ultrasonography is a useful tool for the differential diagnosis of acute flank pain. Renal stones appear as a focal area of echogenicity with acoustic shadowing on ultrasonography. In acute pyelonephritis (APN), the kidneys may be enlarged and have a hypoechoic parenchyma with loss of the normal corticomedullary junction. However, clinical and laboratory correlations are essential for the diagnosis of renal stones and APN through imaging studies. This review describes the typical ultrasonography features of renal stones and APN. Moreover, in daily practice, cross-sectional imaging is essential and widely used to confirm renal stones and APN and to differentiate them from other diseases causing flank pain. Other diseases causing acute flank pain are also described in this review.

Keywords: Ultrasonography; Kidney; Urinary calculi; Pyelonephritis

\section{Introduction}

Acute flank pain due to renal stones or acute pyelonephritis (APN) is a common problem in patients presenting to emergency departments. Radiology plays a vital role in the evaluation of these patients. Several imaging modalities can be used, including ultrasonography (US), computed tomography (CT), conventional radiography, and intravenous urography $[1,2]$. The classic presentation of renal colic is the acute onset of unilateral flank pain with radiation to the groin, dysuria, and hematuria. US can be used to diagnose stones in the renal collecting system when a focal area of echogenicity with acoustic shadowing is identified. When examining the right kidney, the liver can be used as a sonic window, with the patient tilted $15^{\circ}$ to the left. When examining the left kidney, the left lateral decubitus position is helpful. In general, directing the patient to breathe deeply and hold his or her breath for a while will help move the ribs and bowel gases out of the window. As in other US examinations, a high-frequency transducer should be used as long as sufficient penetration is achieved in the US of the kidney, and a convex probe of about $5 \mathrm{MHz}$ is usually used in adults. In order to effectively detect renal stones on US, the ultrasound gain should be adjusted to be slightly lower and the focal zone should be adjusted to be near the suspicious stones. If the focal zone is located slightly deeper than the stones, posterior shadowing is more pronounced and less noise is present within the window.

Associated dilatation of the renal collecting system may occur, depending on the size and location of the stone, the length of time the obstruction has been present, and whether the obstruction is partial or

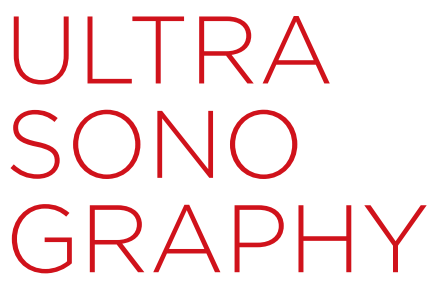

PICTORIAL ESSAY

https://doi.org/10.14366/usg. 17051 pISSN: 2288-5919 • eISSN: 2288-5943 Ultrasonography 2018;37:345-354

Received: July 19, 2017

Revised: October 22, 2017

Accepted: November 26, 2017

Correspondence to:

Ki Choon Sim, MD, Department of Radiology, Korea University Anam Hospital, Korea University College of Medicine, 73 Inchon-ro, Seongbuk-gu, Seoul 02841, Korea

Tel. +82-2-920-5578

Fax. +82-2-929-3796

E-mail: ha2sky@hanmail.net

This is an Open Access article distributed under the terms of the Creative Commons Attribution NonCommercial License (http://creativecommons.org/ licenses/by-nc/3.0/) which permits unrestricted noncommercial use, distribution, and reproduction in any medium, provided the original work is properly cited.

Copyright (C) 2018 Korean Society of Ultrasound in Medicine (KSUM)

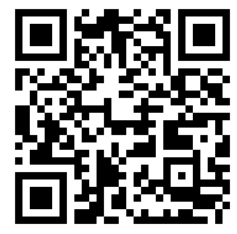

How to cite this article:

Sim KC. Ultrasonography of acute flank pain: a focus on renal stones and acute pyelonephritis. Ultrasonography. 2018 Oct;37(4):345-354. 
complete. If hydronephrosis is suspected, it is necessary to check the fullness of the bladder. If the bladder is full, the kidney should be rechecked after urination to confirm whether the hydronephrosis is due to an overdistended bladder.

In APN, the ultrasonographic appearance of the kidney is usually normal-as are the CT findings of the kidney-but the kidney may be enlarged and have a hypoechoic or hyperechoic parenchyma with loss of the normal corticomedullary junction, abscess formation, or areas of hyperperfusion on color Doppler images [3].

Although US is useful for the diagnosis of renal stones and APN, CT is the main imaging tool that is used, as it provides highly specific findings and an accurate assessment of the renal and extrarenal extent of disease. The purpose of this review is to discuss practical approaches, the findings of US evaluations of acute flank pain, and the pitfalls of US.

\section{Clinical Considerations Regarding Renal Stones}

Renal or ureteric colic is the most common urologic emergency and one of the most common cause of acute abdominal pain. Patients treated for renal stones are usually between 30 and 60 years of age. The disease affects men 3 times as often as it does women [2]. Renal colic manifests as the sudden onset of severe pain radiating to the flank or inguinal area. This severe pain is caused by the passage of a stone formed in the kidney [4]. The passage of stones into the ureter with subsequent acute obstruction, proximal urinary tract dilation, and spasm is associated with classic renal colic. The most useful laboratory finding for renal stones is hematuria on microscopic urinalysis. However, the specificity (48\%) and negative predictive value (65\%) of this finding were found to be low [5]. Thus, imaging studies are essential for diagnosing or excluding renal stones with appropriate consideration of clinical and laboratory correlations.

\section{Ultrasonographic Evaluation of Renal Stones}

US is a widely available imaging modality that does not expose the patient to ionizing radiation. Renal stones on US are hyperechoic and show posterior acoustic shadowing depending on their size and the transducer frequency (Fig. 1). Generally, US is highly effective at showing large stones ( $>5 \mathrm{~mm}$ ), with nearly 100\% sensitivity, but poor at visualizing stones smaller than $3 \mathrm{~mm}[2,6]$. It may be hard to distinguish small stones from vascular calcifications.

When renal stones obstruct the ureter, US is very effective in demonstrating the secondary sign of hydronephrosis. Although US can detect renal stones located at the upper ureter (Fig. 2) or distally at the ureterovesical junction that cause hydronephrosis, most ureteral stones are typically obscured by overlying bowel gas. US had a sensitivity of only $37 \%$ for direct ureteral stone detection, but when hydronephrosis was included as a positive sign for a ureteral stone the sensitivity increased to $74 \%[2,7]$. Additional kidneyureter-bladder plain abdomen radiography or $\mathrm{CT}$ increased the sensitivity for ureteric stones to $100 \%$.

Many grading systems have been developed for hydronephrosis, but there is no specific grading system for hydronephrosis caused by a renal stone in adults. The Society of Fetal Urology classification from grade I to IV is usually used to grade hydronephrosis in adults (Fig. 3) [8]. However, in early stages of ureteral obstruction or when it is improving, hydronephrosis may be absent or only present to a

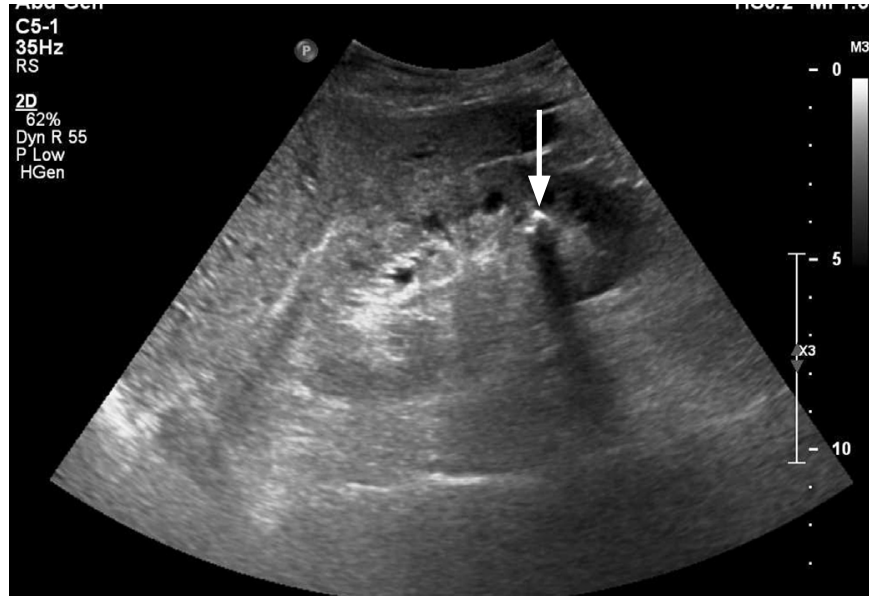

A

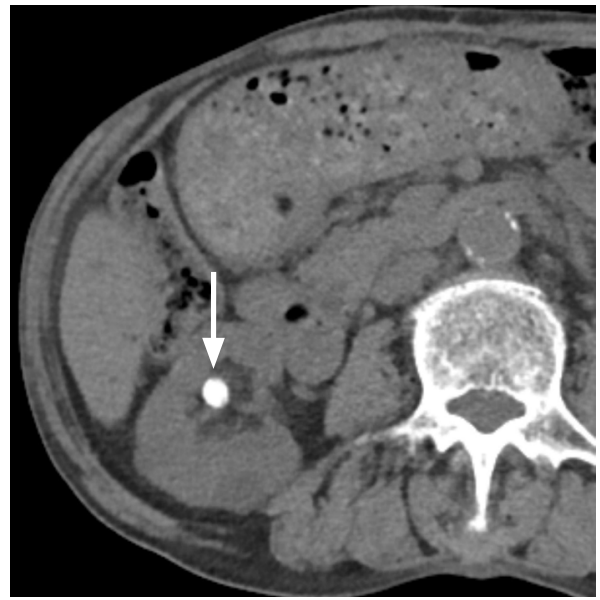

B

Fig. 1. Typical case of a calyceal stone.

A. A sagittal ultrasonography of the right kidney shows a hyperechoic stone (arrow) with posterior acoustic shadowing in the lower pole. B. Axial nonenhanced CT shows a calcified stone (arrow) in the right kidney. 
minimal degree. According to a recent article, up to $11 \%$ of renal stone and colic patients may not have hydronephrosis, and only mild hydronephrosis appears in most of them (up to 71\%) [9]. Therefore, the possibility of false-negative US studies should be kept in mind.

In addition, secondary findings of renal stones or hydronephrosis include the twinkling artifact and the absence of a urine jet on the affected side. The twinkling artifact is a rapid alternation of color immediately behind a stone that may be observed on color Doppler imaging, just like gallbladder stones (Fig. 4A, B) [10]. The twinkling artifact is mainly observed on rough, hyperechoic, irregular surfaces with multiple cracks, which cause a strong reflection of incident ultrasound waves. It appears as a discrete focus of alternating

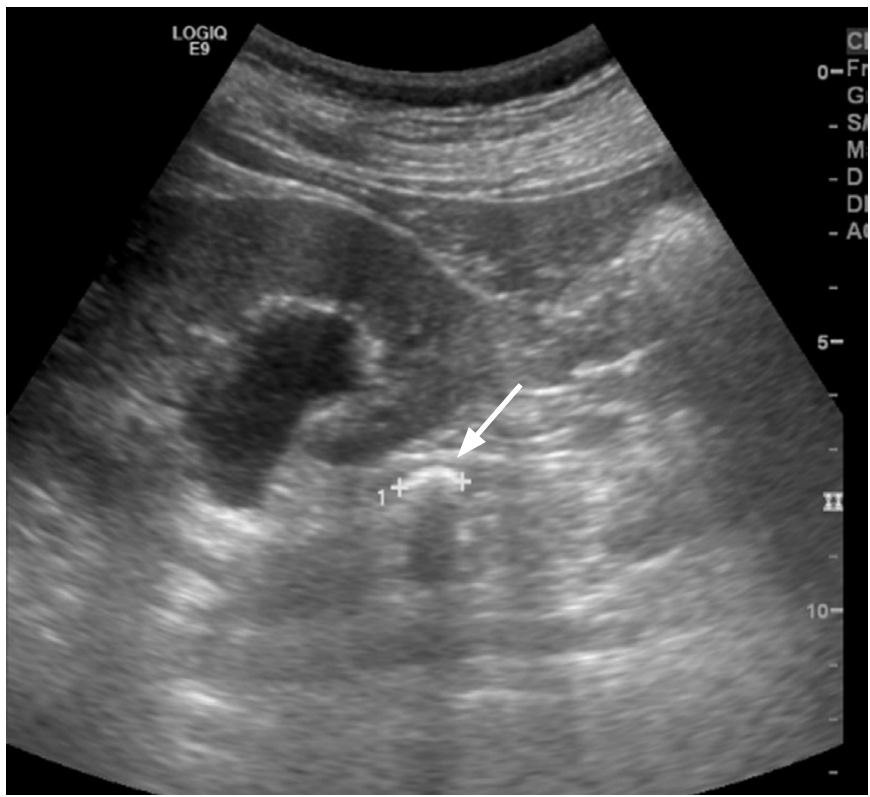

Fig. 2. Upper ureteral stone (arrow) with combined hydronephrosis. Sagittal ultrasonography of the right kidney shows moderate hydronephrosis and dilatation of the proximal ureter due to a hyperechoic stone in the upper ureter.

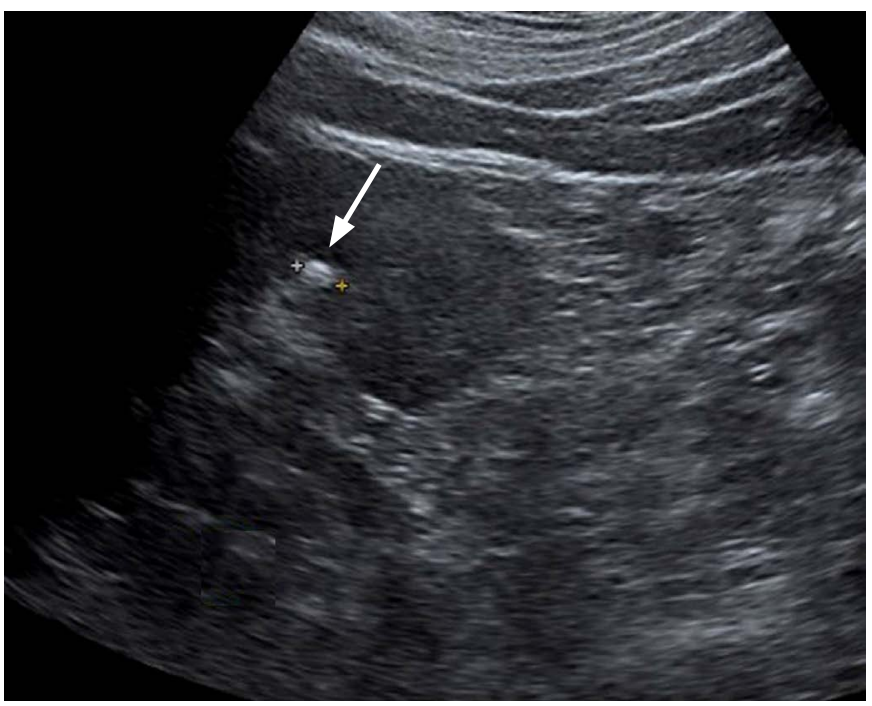

A

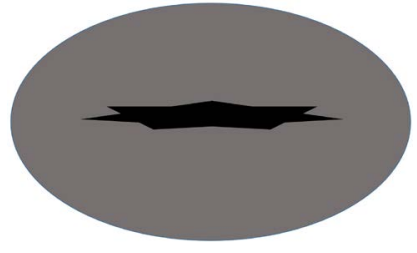

A

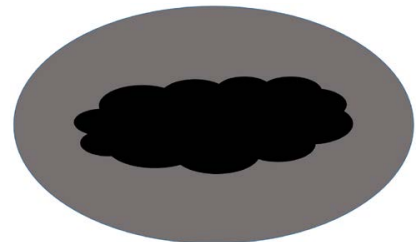

C

Fig. 3. A scheme of different grades of hydronephrosis according to the Society of Fetal Urology classification.

A. Grade 1: renal pelvis is barely dilated without calyceal dilation. B. Grade 2: renal pelvis is further dilated and some calyces may be visualized. C. Grade 3: renal pelvis and minor calyces are diffusely and uniformly dilated; however, renal parenchyma is of normal thickness. D. Grade 4: renal pelvis and minor calyces are severely dilated with thinning of the renal parenchyma.

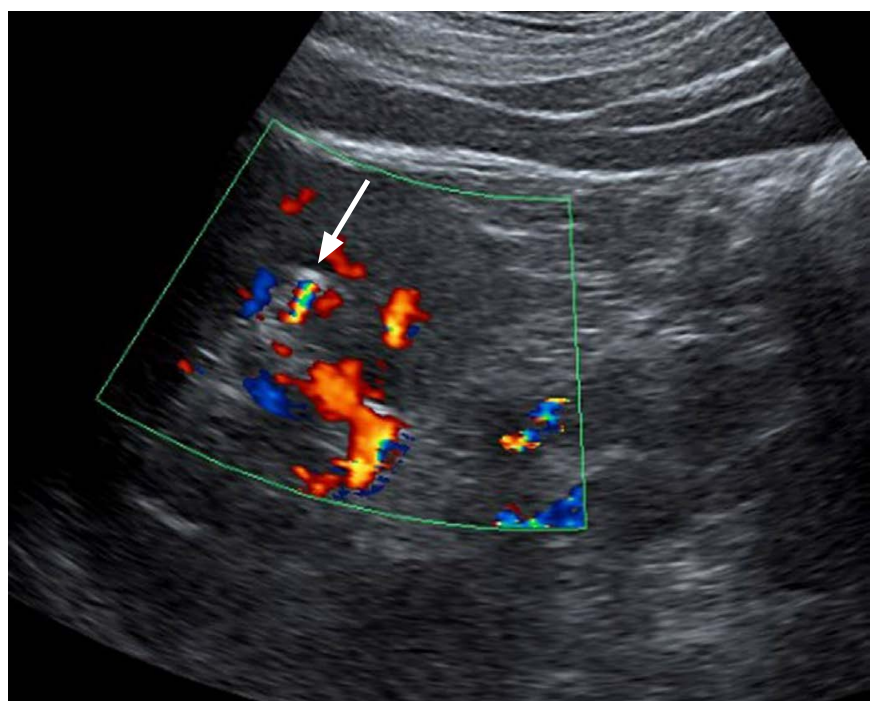

B

Fig. 4. Secondary sign of a renal stone.

A. A grayscale ultrasonography of the left kidney shows an echogenic nonobstructing renal stone (arrow) at the mid-pole. B. Note the twinkling comet tail artifact (arrow) deep to the stone on the color Doppler image. 


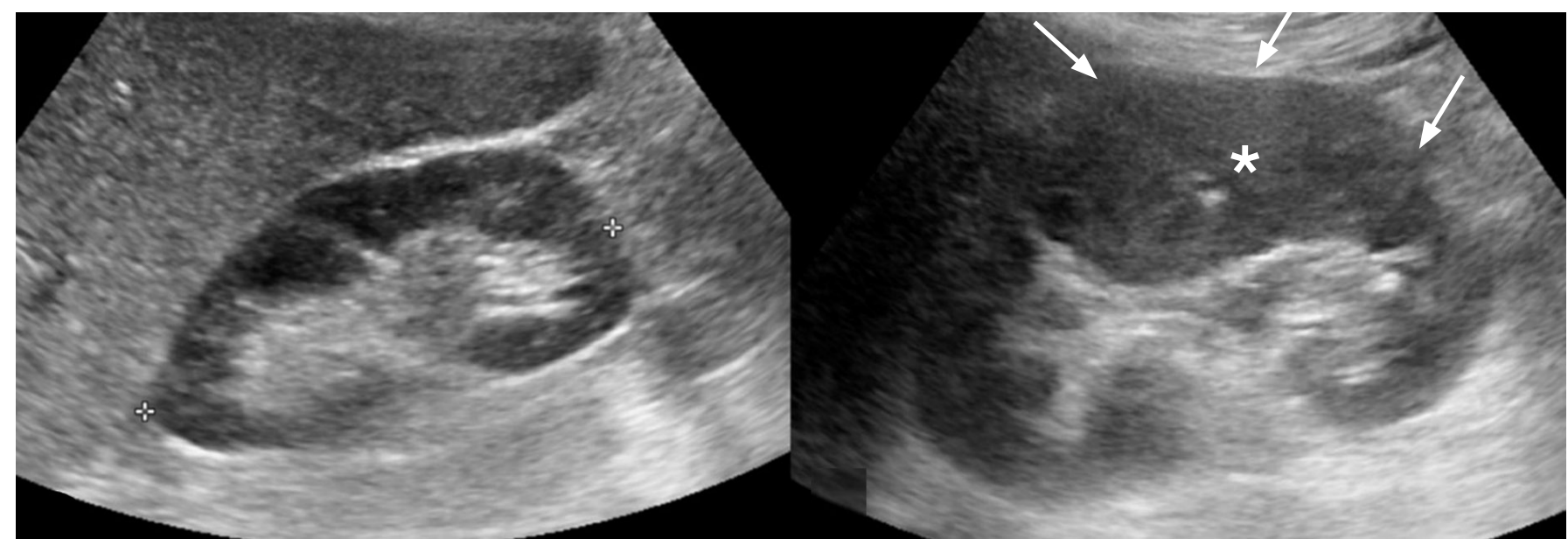

Fig. 5. A 42-year-old woman with left flank pain and fever. Grayscale ultrasonography shows a relatively enlarged left kidney (right panel) with increased cortical echogenicity (arrows and asterisks) compared with the right kidney (left panel). This finding may suggest parenchymal swelling due to inflammation. The urinalysis findings and clinical symptoms were also compatible with acute pyelonephritis.

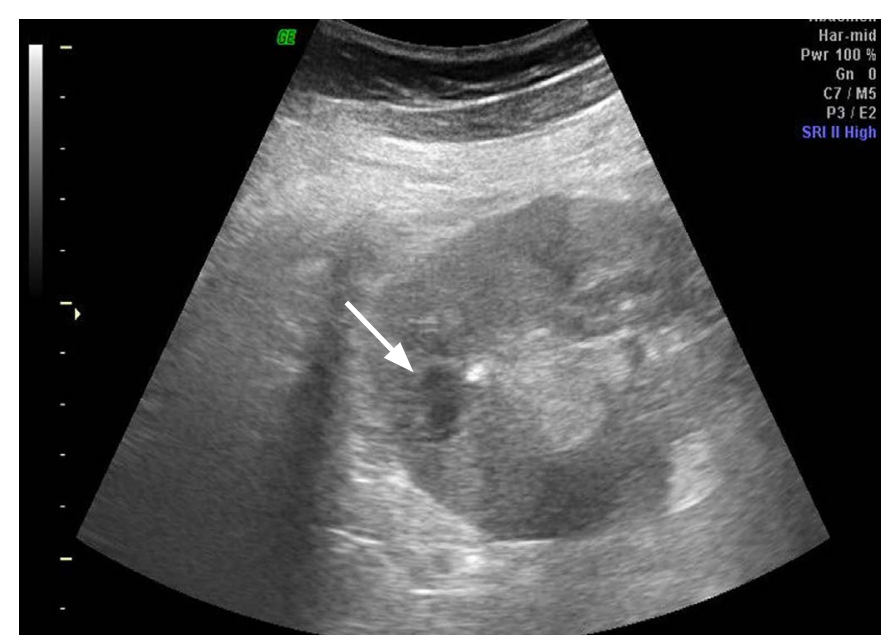

A
Fig. 6. A 58-year-old woman with left flank pain and sustained fever.

During antibiotic treatment for suspected acute pyelonephritis (APN) in a private hospital, she was referred for a sustained fever. A. On a grayscale ultrasonography, the left kidney shows heterogeneously increased cortical echogenicity, and there is a small hypoechoic lesion in the upper pole (arrow). The possible cause was either a small cyst or abscess. B. A CT scan was obtained to differentiate the possibility of abscess. Portal-phase CT shows an irregularly-shaped hypodense lesion (arrows) in the upper pole of the left kidney, which is a finding highly suspicious of an abscess. Additionally, the left kidney shows decreased parenchymal enhancement (white asterisks) compared to the right kidney (black asterisk), and corticomedullary differentiation is also unclear. This finding suggests medical renal disease, such as APN.

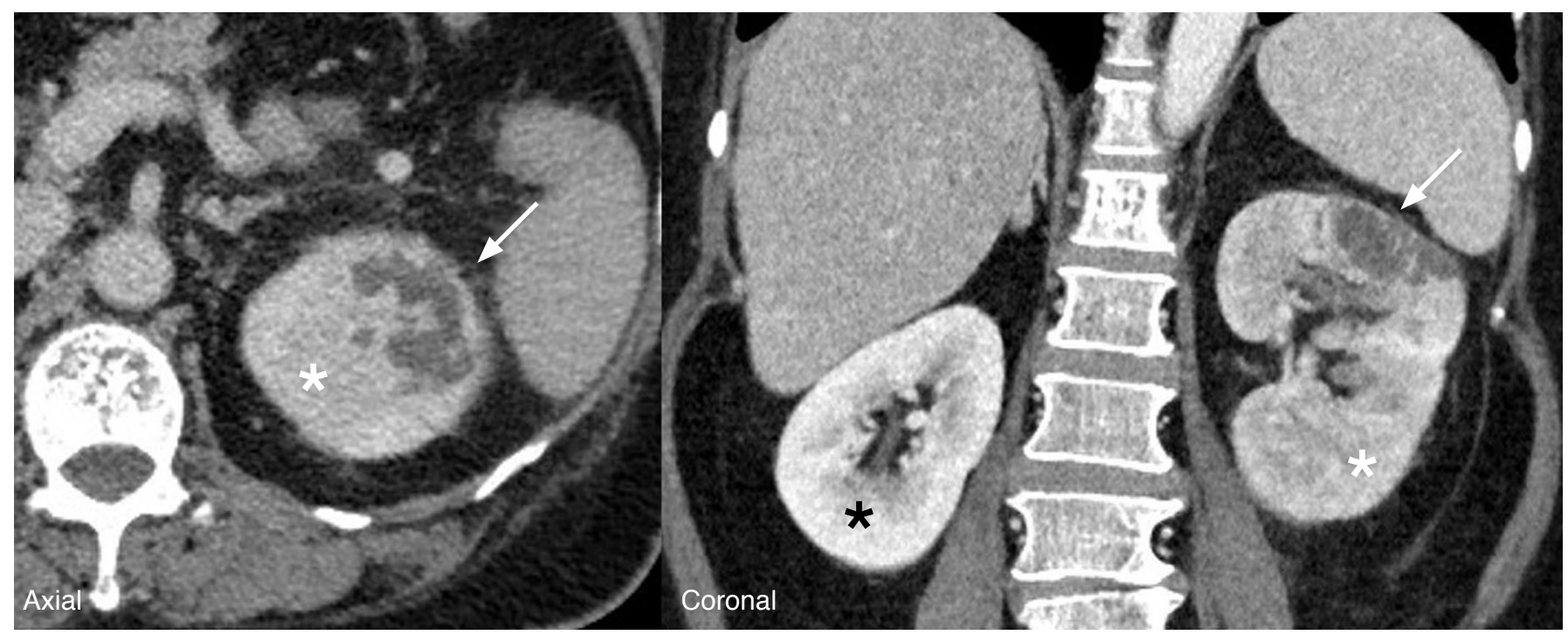

B 
colors with or without an associated color comet-tail artifact [11]. The appearance of the twinkling artifact is highly dependent on the machine settings, and in order to observe it clearly, it is recommended to use a low pulse repetition frequency and high color priority [10]. Urine jets occur from the periodic contraction of the ureters and are easily visible on color Doppler imaging. Obstruction manifests as either the complete absence of the urine jet on the affected side or continuous low-level flow on the affected side, depending on the severity of the obstruction $[2,6]$.

\section{Clinical Considerations Regarding APN}

The diagnosis of APN in adults is usually made by a combination of the typical clinical features of flank pain, elevated body temperature, and dysuria combined with abnormal urinalysis findings such as bacteriuria and/or pyuria. However, routine radiologic imaging is not required for the diagnosis and treatment of uncomplicated cases of APN in adult patients $[3,12]$. A radiologic evaluation may be reserved for patients with atypical symptoms, the elderly, diabetic patients, patients with congenital anomalies, and so on. US is a useful first-line tool for evaluating urinary tract infections without any radiation hazard. However, when acute bacterial pyelonephritis is suspected and an imaging work-up is required, as in complicated cases, CT is a more reasonable modality than US, because complications and possible causes (intraparenchymal or collecting system gas, a small microabscess, perinephric extension of infection, ureter stone, etc.) can be diagnosed accurately. This rationale is consistent with the American College of Radiology Appropriateness Criteria in "variant 2 of clinical condition" [13].

\section{Ultrasonographic Evaluation of APN}

The most common sonographic finding of APN is normal echogenicity. In other words, most patients with clinically suspected APN (up to $80 \%$ ) have negative US results [3]. When positive findings of APN are suspected on US, they can include hypoechogenicity due to parenchymal edema and hyperechogenicity in cases of hemorrhage, swelling (Fig. 5), a perfusion defect on power Doppler images, loss of corticomedullary differentiation,

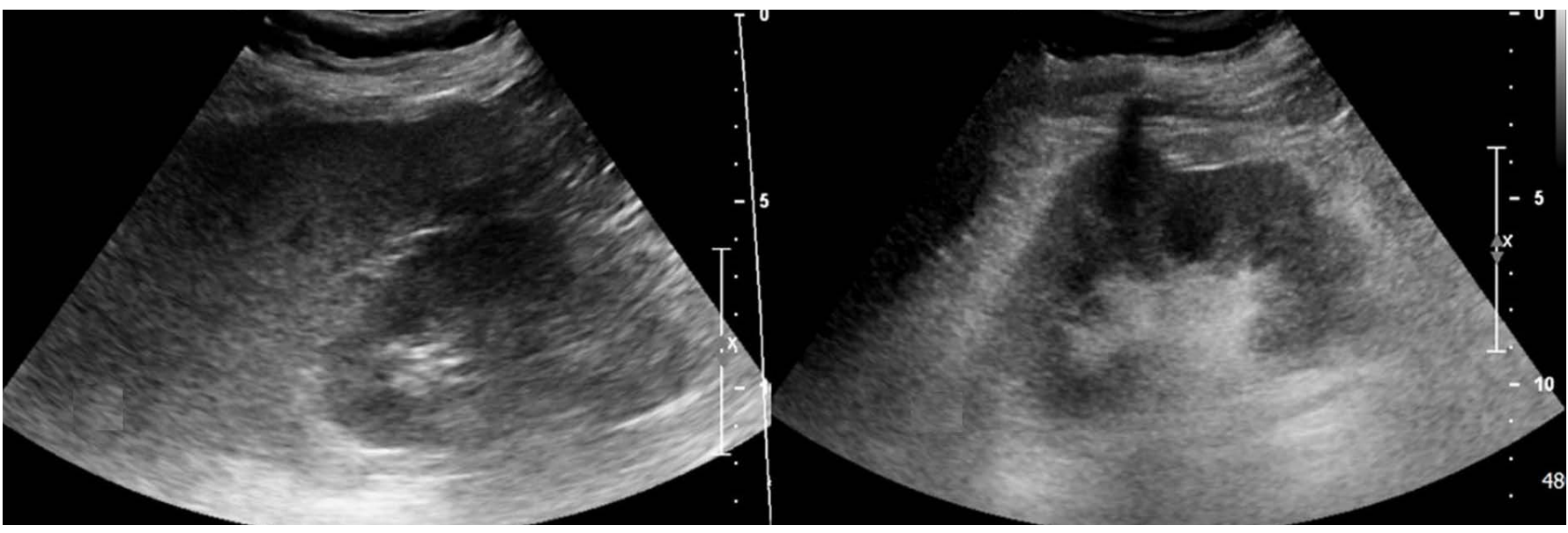

A

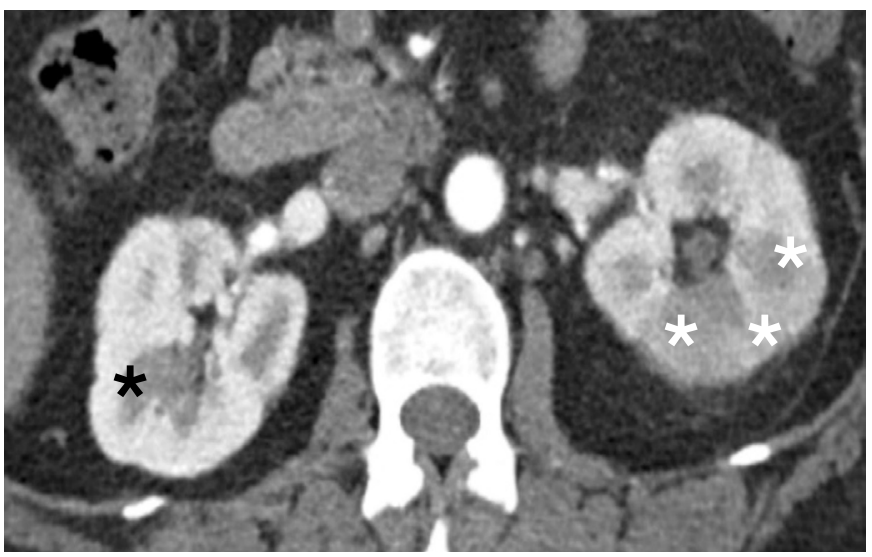

B
Fig. 7. A 53-year-old woman with left flank pain.

A. On grayscale ultrasonography, it is difficult to distinguish the echogenicity difference of the right (left panel) and left kidney (right panel). B. Enhanced CT demonstrates ill-defined hypodense lesions in the left kidney (white asterisks), suggesting acute pyelonephritis. The right kidney shows normal parenchymal enhancement and corticomedullary differentiation (black asterisk). Thus, CT is considered to be the modality of choice for evaluating acute bacterial pyelonephritis. 
wall thickening of the renal pelvis, or abscess formation (Fig. 6A, B) $[3,14]$. Despite the presence of several ancillary findings for the diagnosis of APN, it can be very difficult to differentiate between artifacts and true positive findings if the sonic window is poor (due to bowel gas, bony thorax, or thick subcutaneous fat tissue) or if the patient's respiration is irregular (due to conditions such as tachypnea) (Fig. 7A, B). Thus, CT is considered to be the modality of choice for evaluating acute bacterial pyelonephritis. Another advantage of $\mathrm{CT}$ is that it can provide comprehensive anatomic and physiological information, thereby helping to accurately characterize both intrarenal and extrarenal pathologic conditions (Fig. 8A, B).
Mimics of Renal Colic

\section{Unidentified bright objects}

On US, tiny echogenic foci are occasionally seen in the parenchyma or hilum. These are called unidentified bright objects (UBO), and these echogenic foci frequently accompany the reverberation artifact, but posterior shadowing is absent. The possible causes of UBOs in the kidney are tiny stones, tiny cysts, small calyceal diverticulum areas with wall calcification or milk of calcium, calcified arteries, and tiny angiomyolipomas (Fig. 9).

\section{Medullary nephrocalcinosis}

On US, there are many causes of altered renal parenchymal echogenicity. The ultrasonographic appearance of medullary

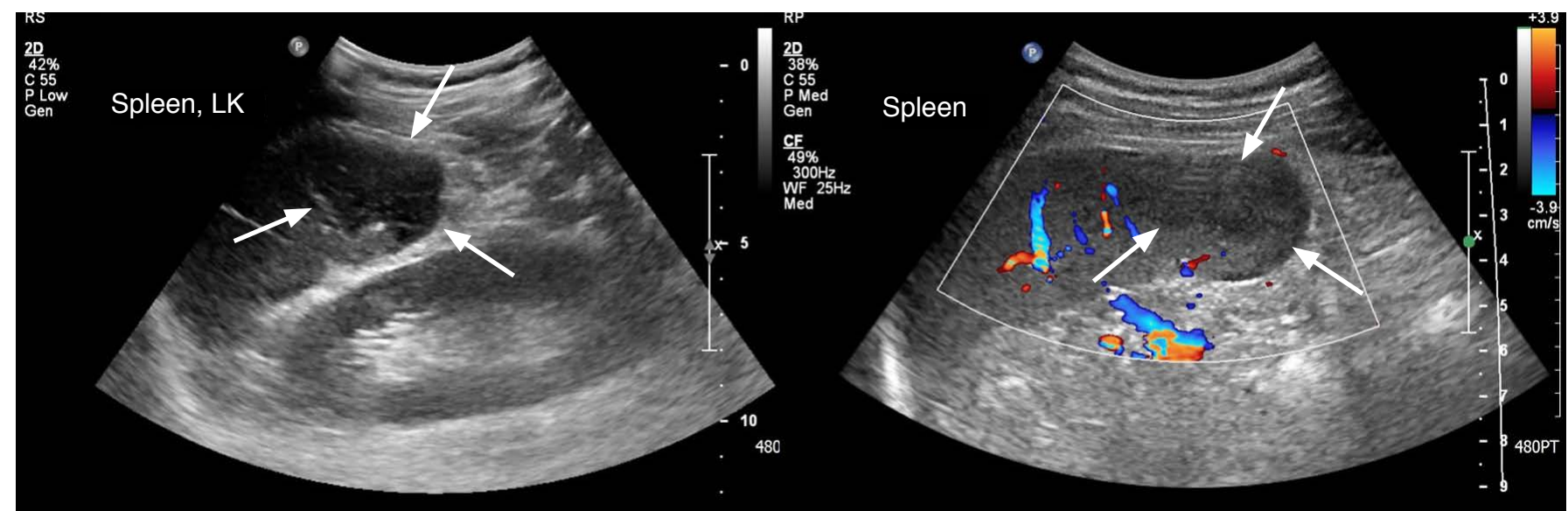

A

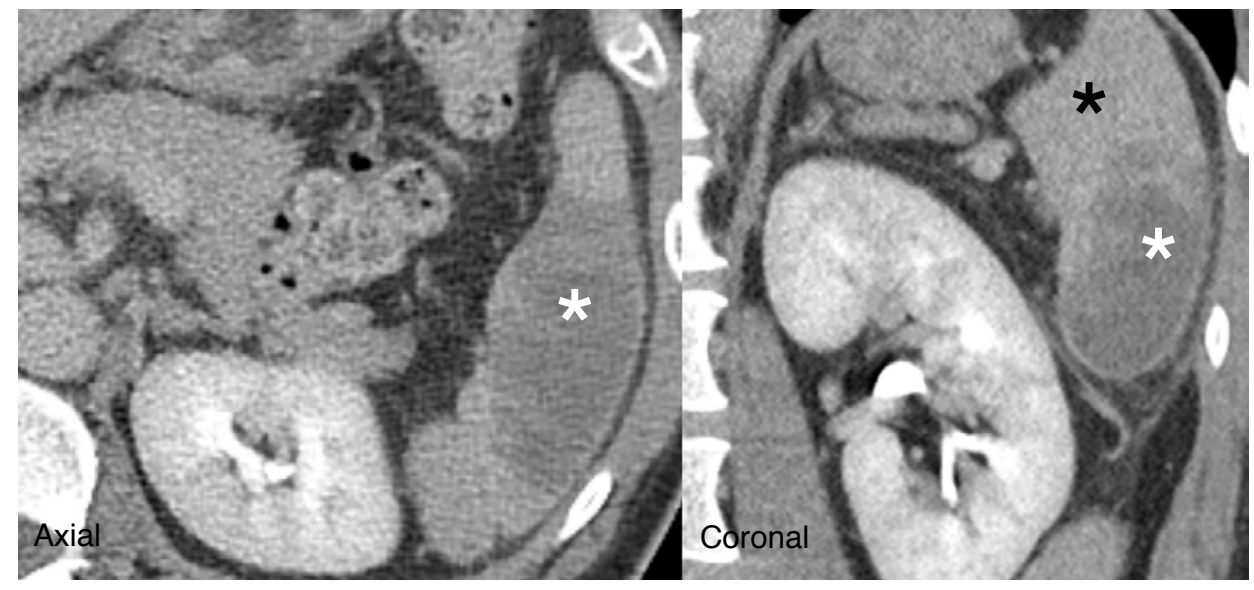

$\mathrm{B}$

Fig. 8. A 44-year-old man with left flank pain and leukocystosis.

A. This patient visited the emergency department due to acute left flank pain. On grayscale ultrasonography, there were no remarkable findings in the left kidney. However, hypoechoic areas are seen in the spleen (arrows). On color Doppler ultrasonography, there is an area of decreased perfusion. B. On enhanced CT, there is a hypoattenuating area in the spleen (white asterisks) compared to the normal parenchyma (black asterisk). This finding is compatible with focal splenic infarction without a demonstrable cause. Splenic infarction is a result of ischemia to the spleen but usually requires no treatment. 
nephrocalcinosis is characterized by echogenic materials with a variable degree of acoustic shadowing in the region of the renal pyramids (Fig. 10). Depending on the degree of calcium deposition in the renal pyramid, the CT density (Hounsfield units) may also vary [15]. Medullary nephrocalcinosis occurs in various diseases as a non-specific renal manifestation. In individual cases, it is difficult

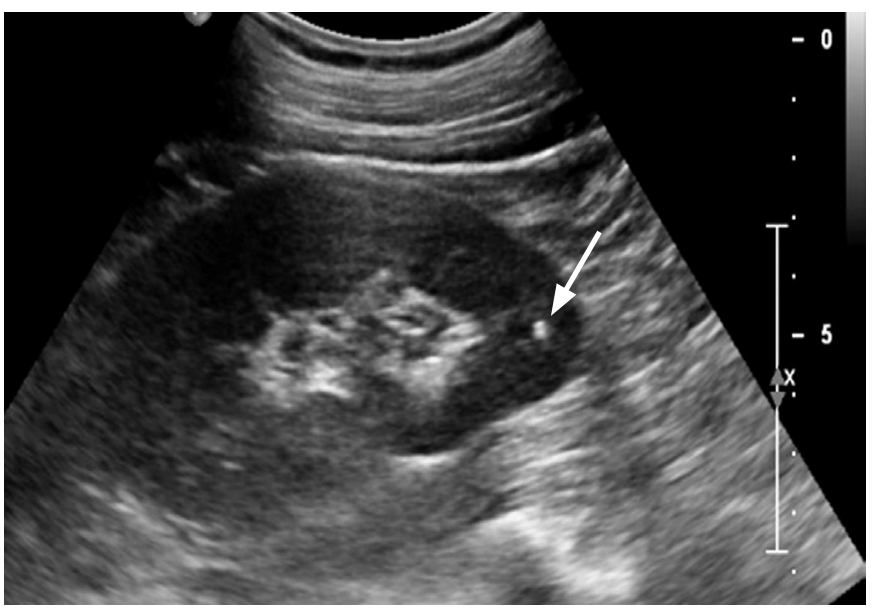

Fig. 9. Unidentified bright objects in the kidney parenchyma. Tiny echogenic foci (arrow) are occasionally seen in the parenchyma on ultrasonography. They are called unidentified bright objects (UBOs). These echogenic foci frequently accompany the reverberation artifact, but posterior shadowing is absent. The possible causes of UBOs in the kidney are tiny stones, tiny cysts, small calyceal diverticulum areas with wall calcification or milk of calcium, calcified arteries, and tiny angiomyolipomas. However, UBOs observed on ultrasonography may not be clearly observed on computed tomography.

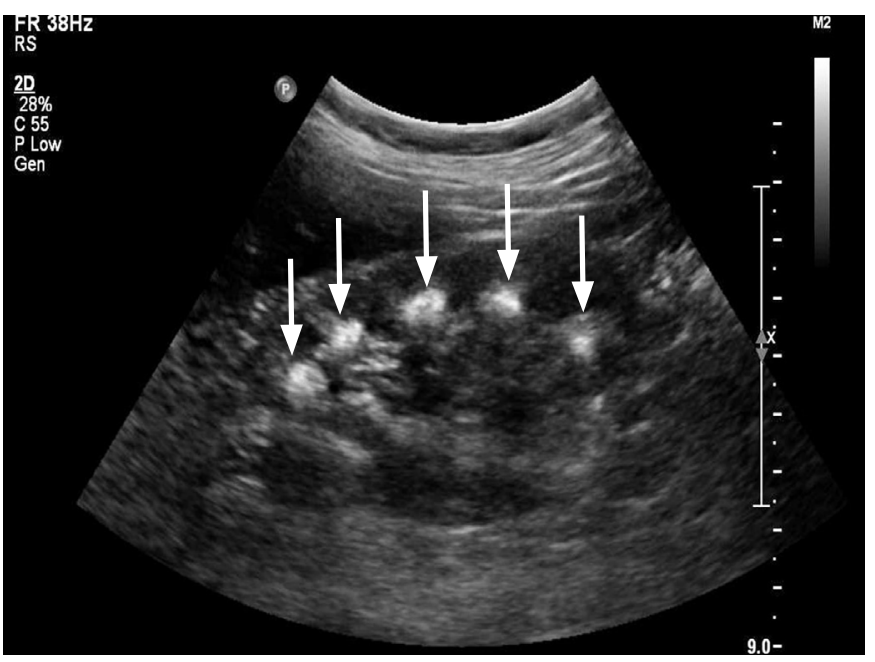

A

Fig. 10. A 56-year-old woman with a medullary sponge kidney.

A. Ultrasonography shows increased echogenicity of the renal medulla (the pyramids are normally hypoechoic to the cortex). This appearance is typical of medullary nephrocalcinosis (arrows). B. Color Doppler ultrasonography shows twinkling artifacts (arrows) at the renal medulla.

e-ultrasonography.org

Ultrasonography 37(4), October 2018 


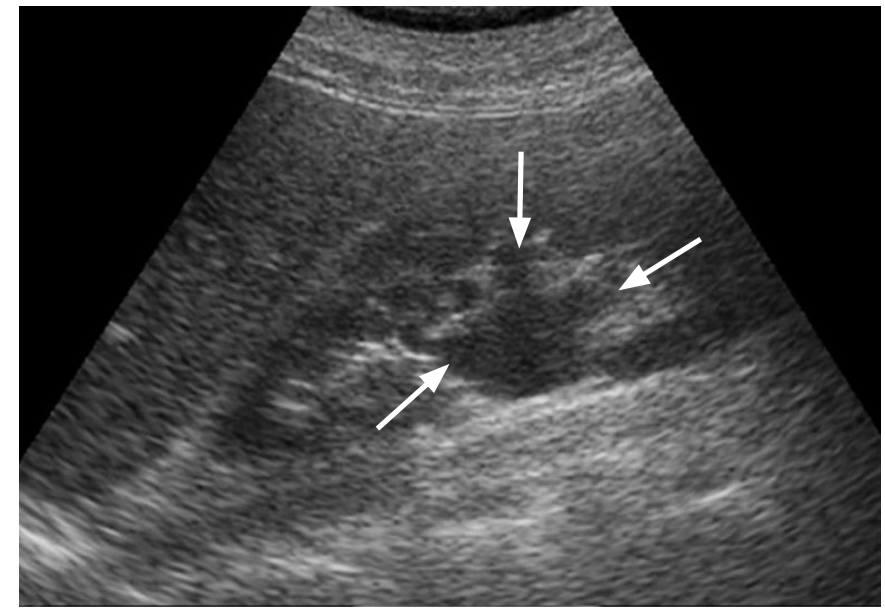

A

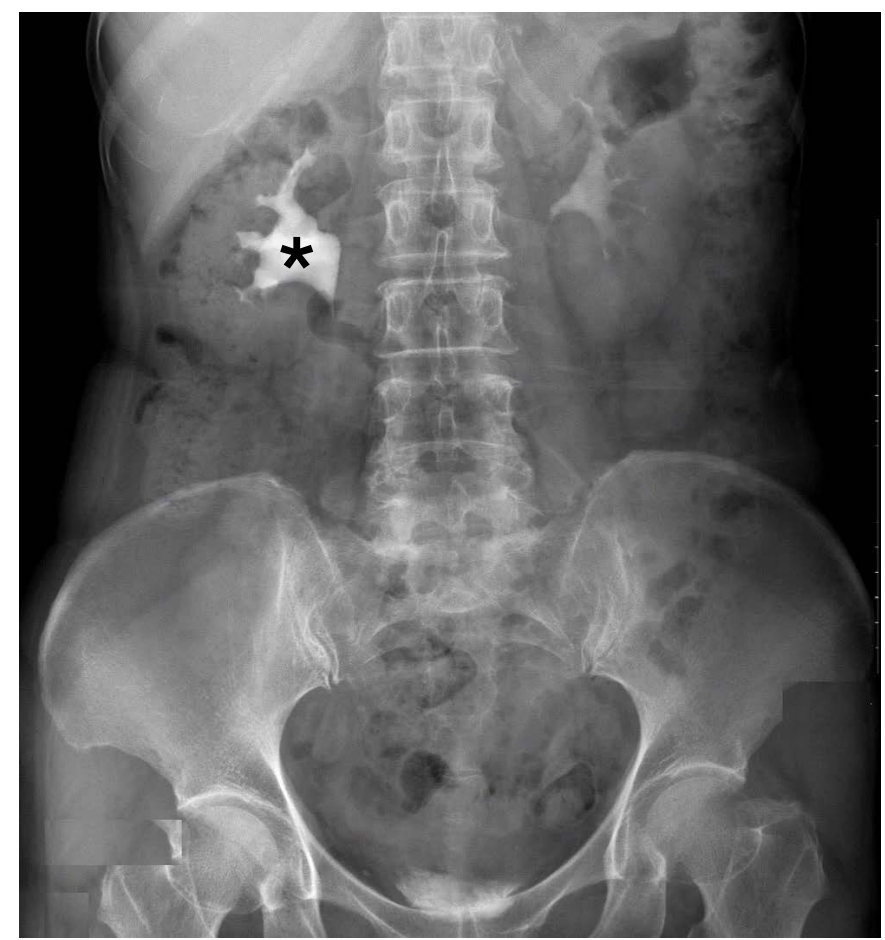

C

\section{Conclusion}

Although US has several limitations in adults with acute flank pain, it is a useful modality to diagnose stones and to confirm the occurrence of complications of APN, so it is important to understand these characteristic findings and other diseases that mimic them. In addition, other imaging modalities such as $\mathrm{CT}$ can be recommended

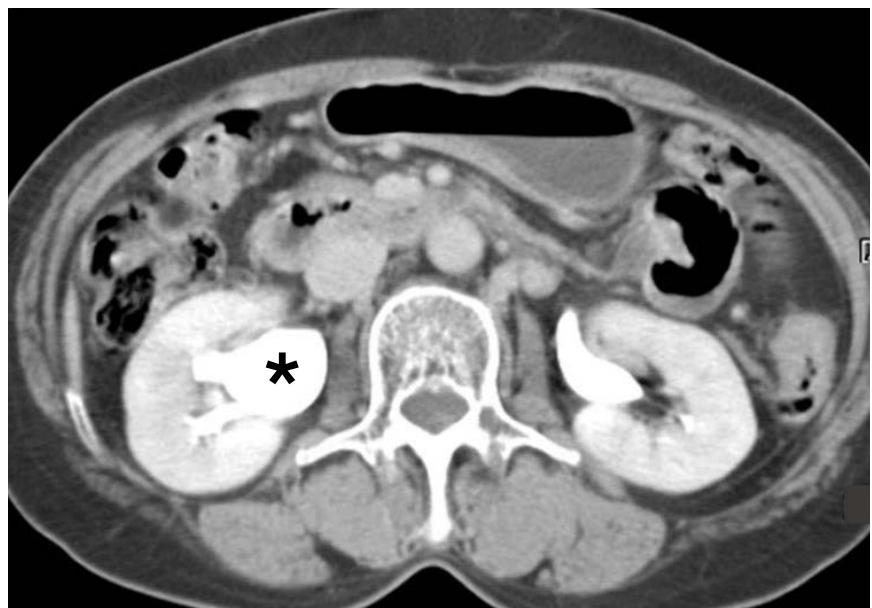

B

Fig. 11. Extrarenal pelvis.

A. Grayscale ultrasonography of the right kidney shows dilatation of the renal pelvis (arrows). A beginner could mistakenly think that mild hydronephrosis was present. B, C. A prominent extrarenal pelvis (asterisks) is a well-known normal variation that mimics hydronephrosis on excretory axial CT. (B) and intravenous pyelography (C). Note that neither calyceal blunting on an intravenous pyelogram nor contrast excretion delay of the right kidney is present.

if the clinical or radiological diagnosis is ambiguous.

\section{Conflict of Interest}

No potential conflict of interest relevant to this article was reported.

ORCID: Ki Choon Sim: http://orcid.org/0000-0002-3344-8018 


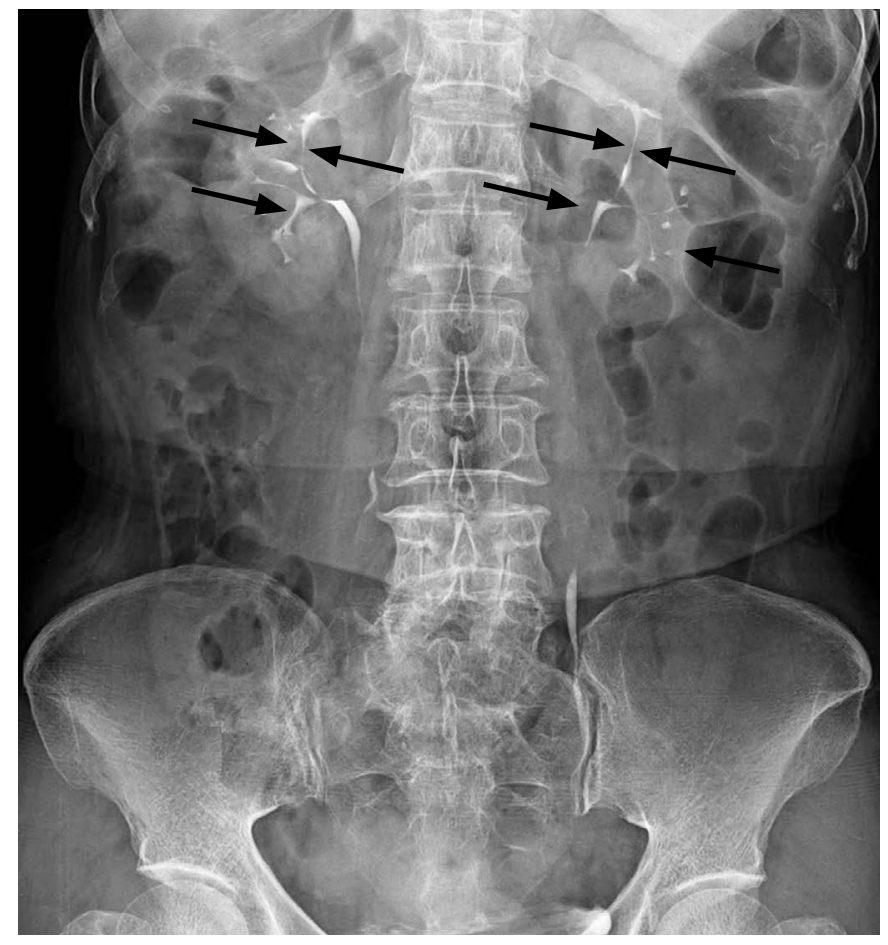

A
Fig. 12. Parapelvic cysts.

A. On an intravenous pyelogram, stretching and compression of the calyces (arrows) are noted in both kidneys. B. Grayscale ultrasonography shows dilatation of the renal pelvis and calyces (asterisks) in both kidneys mimicking bilateral hydronephrosis. C. Excretory coronal CT shows multiple cystic lesions (asterisks) in the bilateral renal hila with intervening normal renal pelvis and calyces.

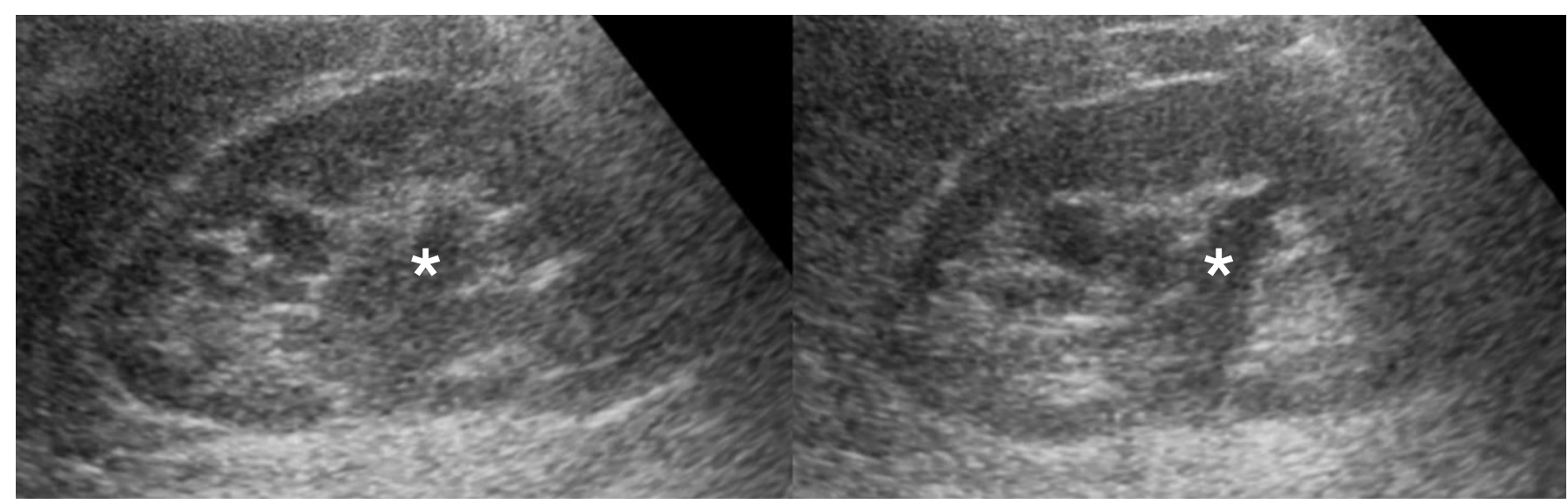

B

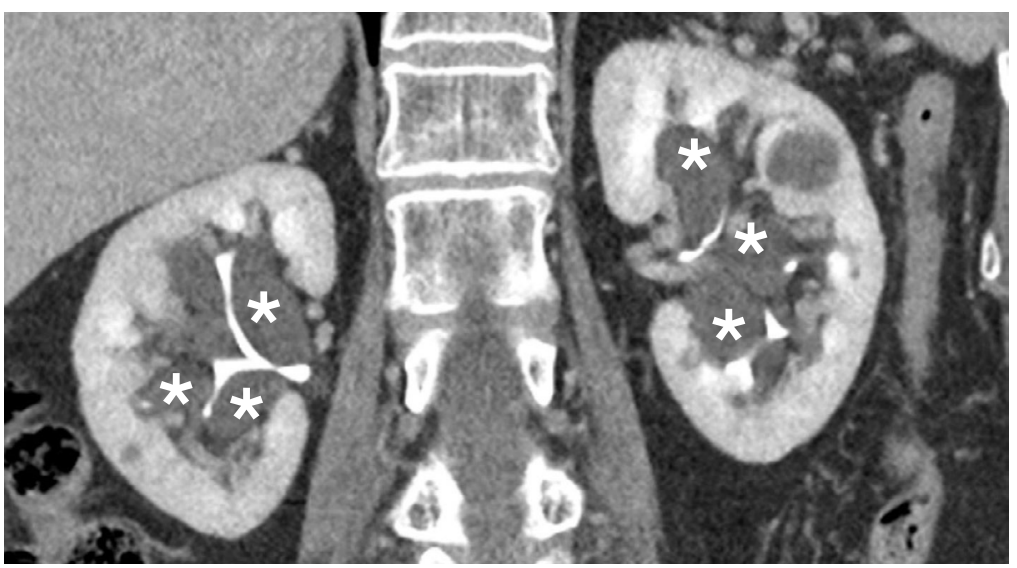

C 


\section{References}

1. Hill MC, Rich JI, Mardiat JG, Finder CA. Sonography vs. excretory urography in acute flank pain. AJR Am J Roentgenol 1985; 144:1235-1238.

2. Tamm EP, Silverman PM, Shuman WP. Evaluation of the patient with flank pain and possible ureteral calculus. Radiology 2003;228:319329.

3. Craig WD, Wagner BJ, Travis MD. Pyelonephritis: radiologicpathologic review. Radiographics 2008;28:255-277.

4. Gaspari RJ, Horst K. Emergency ultrasound and urinalysis in the evaluation of flank pain. Acad Emerg Med 2005;12:1180-1184.

5. Luchs JS, Katz DS, Lane MJ, Mellinger BC, Lumerman JH, Stillman $C A$, et al. Utility of hematuria testing in patients with suspected renal colic: correlation with unenhanced helical CT results. Urology 2002;59:839-842.

6. Moore $\mathrm{CL}$, Scoutt L. Sonography first for acute flank pain? J Ultrasound Med 2012;31:1703-1711.

7. Aslaksen A, Gothlin JH. Ultrasonic diagnosis of ureteral calculi in patients with acute flank pain. Eur J Radiol 1990;11:87-90.

8. Fernbach SK, Maizels M, Conway JJ. Ultrasound grading of hydronephrosis: introduction to the system used by the Society for Fetal Urology. Pediatr Radiol 1993;23:478-480.

9. Song Y, Hernandez N, Gee MS, Noble VE, Eisner BH. Can ureteral stones cause pain without causing hydronephrosis? World J Urol 2016;34:1285-1288.
10. Kamaya A, Tuthill T, Rubin JM. Twinkling artifact on color Doppler sonography: dependence on machine parameters and underlying cause. AJR Am J Roentgenol 2003;180:215-222.

11. Dillman JR, Kappil M, Weadock WJ, Rubin JM, Platt JF, DiPietro MA, et al. Sonographic twinkling artifact for renal calculus detection: correlation with CT. Radiology 2011;259:911-916.

12. Stunell H, Buckley O, Feeney J, Geoghegan T, Browne RF, Torreggiani WC. Imaging of acute pyelonephritis in the adult. Eur Radiol 2007;17:1820-1828.

13. Sandler CM, Amis ES Jr, Bigongiari LR, Bluth El, Bush WH Jr, Choyke $\mathrm{PL}$, et al. Imaging in acute pyelonephritis. American College of Radiology. ACR Appropriateness Criteria. Radiology 2000;215 Suppl:677-681.

14. Lee HJ, Kim YJ, Park HS, Jeon HJ, Park HK, Paick SH, et al. Diagnostic performance of gray-scale sonographic findings for the detection of acute pyelonephritis. J Korean Soc Ultrasound Med 2012;31:35-41.

15. Boyce AM, Shawker TH, Hill SC, Choyke PL, Hill MC, James R, et al. Ultrasound is superior to computed tomography for assessment of medullary nephrocalcinosis in hypoparathyroidism. J Clin Endocrinol Metab 2013;98:989-994.

16. Riehl J, Schneider B, Bongartz D, Sieberth HG. Medullary nephrocalcinosis: sonographic findings in adult patients. Bildgebung 1995;62:18-22.

17. Tarzamni MK, Sobhani N, Nezami N, Ghiasi F. Bilateral parapelvic cysts that mimic hydronephrosis in two imaging modalities: a case report. Cases J 2008;1:161. 